\title{
Screening for ovarian cancer: progress and challenges
}

\author{
Karim Elmasry
}

\section{Introduction}

Ovarian cancer is one of the most aggressive gynaecological malignancies, the high mortality being a direct result of the advanced stage of the disease at the time of diagnosis. However, survival rates of greater than $90 \%$ have been reported with Stage I disease. Consequently there is considerable interest in the development of screening methods for the early detection of ovarian cancer. In the second of two reviews I discuss the challenges of screening for ovarian cancer, the screening tests that are available, the target populations and the screening trials currently in progress.

\section{Rationale for ovarian cancer screening}

Primary carcinoma of the ovary is the leading cause of death from gynaecological malignancy in the Western world. In the UK, it is the fourth most common cause of cancer death among women: there were almost 7000 new cases reported in 2002 (which equates to a lifetime risk of 1 in 48) and there were 4600 deaths from the disease in 2003. ${ }^{1}$

Despite recent advances in surgical techniques and novel chemotherapeutic agents, mortality from ovarian cancer has changed little in the last decade. It is thought that the advanced stage at presentation is primarily responsible for the poor prognosis associated with ovarian cancer. Because it is associated with vague and nonspecific symptoms, about $75 \%$ of women with ovarian cancer will have advanced stage disease (Stage III or IV) at the time of diagnosis. ${ }^{2}$ Despite aggressive treatment, the 5year survival for advanced stage disease is only 30\% compared to survival rates of $60-90 \%$ for early disease (Stage I or II), depending on the degree of tumour differentiation (Figure 1). ${ }^{2}$ Thus there is considerable interest in developing screening methods that detect ovarian cancer at an earlier stage when treatment is more effective and mortality may be reduced.

\section{Challenges of ovarian cancer screening}

Screening for ovarian cancer, however, presents a number of challenges: little is known of the natural history of the disease, a premalignant precursor lesion has yet to be identified, and diagnosis requires surgery in the form of a laparotomy or a laparoscopy. In addition, the incidence of the disease is low, with a lifetime risk estimated to be $1.8 \%$. Thus there is a considerable risk that the morbidity associated with surgery for a false-positive screen will outweigh the potential benefits of screening. It widely accepted that in order to be acceptable a screening test for ovarian cancer must achieve a positive predictive value of

J Fam Plann Reprod Health Care 2006; 32(3): 173-175 (Accepted 6 June 2006)

Translational Research Laboratories, Department of Gynaecological Oncology, Windeyer Institute, London, UK Karim Elmasry, BSc, MRCOG, Clinical Research Fellow

Correspondence to: Dr Karim Elmasry, Translational Research Laboratories, Department of Gynaecological Oncology, Windeyer Institute, Cleveland Street, London W1T 4JF, UK

Tel: +44 (0) 207679 9329. Fax: +44 (0) 2076799243.

E-mail: k.elmasry@ucl.ac.uk at least $10 \%$ (i.e. a maximum of 10 surgical procedures for every cancer identified). In order to achieve this target the test must have a minimum specificity of $99.6 \% .^{3}$ Thus appropriate screening tests must be selected on appropriate target populations to balance the benefits and costs of screening.

\section{Target population}

There are two main target populations that are at risk of ovarian cancer: women in the general population and women at high risk of ovarian cancer by virtue of their family history of the disease.

Ovarian cancer incidence increases with age: over $70 \%$ of cases occur after the age of 50 years, with a maximum incidence of 60.5 per 100000 in the 75-79 years age group. ${ }^{4}$ Some $90 \%$ of women with the disease will have no family history of it. ${ }^{5}$ Thus screening programmes directed at all postmenopausal women are most likely to have the biggest impact on mortality.

Approximately $10 \%$ of ovarian carcinomas have a hereditary basis, the majority caused by a mutation in one of the cancer predisposing genes, BRCA1 and BRCA2. The average cumulative risks by the age of 70 years for ovarian cancer are $39 \%(18-54 \%)$ in BRCA1 mutation carriers and $11 \%(2.4-19 \%)$ in BRCA2 carriers. ${ }^{6}$ Compared to sporadic disease, familial ovarian cancer is often diagnosed in younger women at a later, less curable stage of disease. Thus devising early detection strategies is particularly important in this group of women. However, screening of high-risk women is associated with two problems: high false-negative and false-positive rates. ${ }^{7}$ As many of the women in this group are premenopausal, a higher screen false-positive rate is associated with physiological conditions such as ovulation and common benign conditions. The higher screen false-negative rate is due to the inability to detect multifocal peritoneal disease, which may be associated with familial ovarian cancer.

\section{Screening tests}

A number of screening tests have been or are currently being evaluated, including pelvic examination, ultrasound examination and the measurement of various circulating proteins.

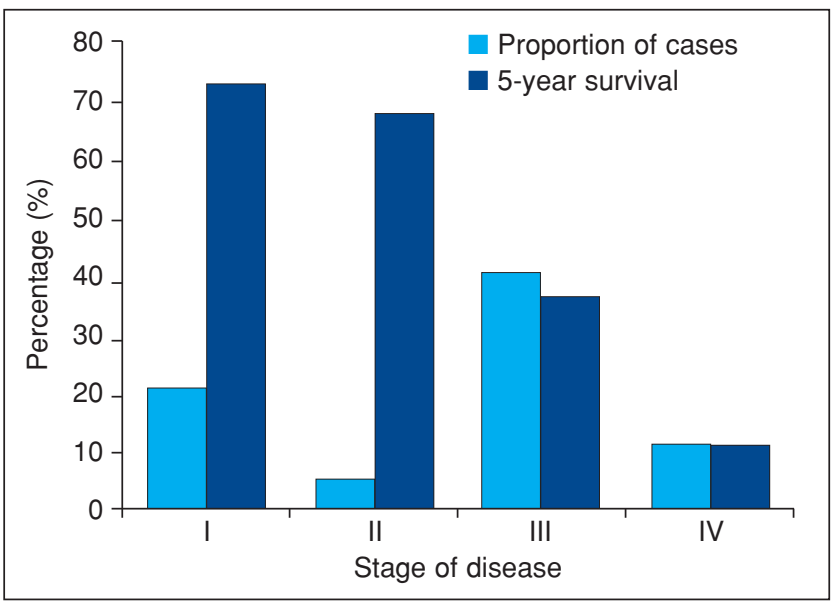

Figure 1 Ovarian cancer: proportion of patients presenting and 5-year survival by stage of disease (data from the Surveillance Epidemiology and End Results Program of the National Cancer Institute ${ }^{2}$ ) 


\section{REVIEW}

\section{Pelvic examination}

Bimanual pelvic examination of asymptomatic women has been disappointing in the early detection of ovarian cancer, lacking sufficient sensitivity and specificity. Andolf et al. reported that in one patient with ovarian cancer, two with borderline tumours and 18 of 24 patients with benign ovarian cysts, the bimanual examination was said to be normal prior to the pelvic ultrasound scan. ${ }^{8}$

\section{Ultrasonography}

Ultrasonographic imaging of the ovaries allows the detection of ovarian enlargement and morphological abnormalities. Initial investigations using the transabdominal approach lacked sufficient specificity due to difficulties in imaging the pelvic organs through the abdominal wall and because of the poor resolution of the older machines used in these initial studies. 9 These problems have been addressed by transvaginal ultrasonography (TVS), which is used with the intention of detecting the earliest morphological changes in the ovary. TVS has demonstrated encouraging sensitivity in the early detection of ovarian cancer but specificity continues to be limited. In a review of results from early studies, Karlan reported sensitivity as high as $100 \%$ and specificity of approximately $98 \% .{ }^{10}$ Improvements in the specificity of TVS can be achieved by the use of a morphology index or through the use of colour Doppler but usually at the cost of some reduction in sensitivity. 11,12

\section{Tumour markers}

CA125, the most widely investigated ovarian tumour marker, is a high molecular weight glycoprotein that is detected in the serum. Using a cut-off threshold level of 30-35 IU/1, approximately $90 \%$ of all advanced ovarian cancers and $50 \%$ of cases with disease confined to the ovaries will have a level exceeding this threshold. ${ }^{13}$ However, specificity is poor as levels can be elevated by a number of other conditions - both benign and malignant such as pregnancy, endometriosis, fibroids and breast cancer, as well as conditions that promote any type of peritoneal irritation. ${ }^{14}$ Using CA125 alone in women aged 50 years or over, a positive predictive value of $4.6 \%$ was achieved in a study of 4290 volunteers. ${ }^{15}$ However, improvements in the sensitivity and specificity of the test can be made by studying the woman's age-related risk in combination with the rate of rise of CA125 rather than the absolute level. The use of a computerised algorithm 'risk of ovarian cancer' (ROC) based on the Bayes' theorem, which compares each individual's pattern of CA125 to the pattern in controls (where levels are static or fall with time) and the pattern in cancers (where the levels rise), has led to an improved specificity, sensitivity and a positive predictive value for predicting the $\mathrm{ROC}$ in the year following the previous screen. ${ }^{16}$

\section{Screening strategies}

When used alone, none of the available tests achieves the specificity and sensitivity required. However, superior specificity and positive predictive values have been achieved with sequential, multimodal screening in the general population

In a recent pilot randomised control trial (RCT) in the UK, 22000 women aged 45 years or older were randomised to routine pelvic examinations or screening. ${ }^{17}$ Screening involved annual CA125 measurements with TVS if the CA125 was $\geq 30$. Although the study did not achieve statistical significance the results were encouraging, with improved survival in women diagnosed with ovarian cancer in the screened group compared to the control group (72.9 vs. 41.8 months, respectively) suggesting that a multimodal approach may be efficacious. In this study, lead time bias was eliminated by measurement of survival from randomisation rather than diagnosis.

In a 10-year ovarian cancer screening trial in Japan, $58.8 \%$ of cancers diagnosed after the introduction of ultrasound screening were Stage I compared to $29.7 \%$ before. ${ }^{18}$

A possible survival benefit was also observed in another study involving 15000 women where the 5-year survival of patients with screen-detected ovarian cancer was $83.6 \pm$ $10.8 \% .12$ However, the lack of a control group in this study raises the possibility of a 'healthy volunteer' bias.

Recently, Olivier et al. reported that in a series of 312 high-risk women screened with CA125 and TVS, screening was not effective as the cancers associated with either an abnormal ultrasound scan or a raised CA125 were found in patients with high-stage disease. 19 High specificity, sensitivity and positive predictive values were achieved. However, the study was limited by the small number of ovarian cancer cases.

\section{Current screening trials}

There are currently two large trials in progress to assess the impact of screening on ovarian cancer in the general population: the Prostate, Lung, Colon and Ovary (PLCO) trial in the USA and the UK Collaborative Trial of Ovarian Cancer Screening (UKCTOCS). In order to assess the impact of screening on ovarian cancer in high-risk women, the UK Familial Ovarian Cancer Screening Study (UKFOCSS) is currently underway.

The PLCO Trial has completed enrolling 74000 women aged 55-74 years. In this RCT, TVS and CA125 are being used together annually as a first-line screen for 3 years and CA 125 alone for a further 2 years. ${ }^{20}$ Women are randomised either to the interventional arm with annual screening or to an observational standard care control arm. A positive result in either of the screening tests results in a gynaecological referral. Follow-up is for 13 years from the point of randomisation, with the endpoint of the study being the health status and/or cause of death.

UKCTOCS is an RCT in which 200000 postmenopausal women are randomised into one of three treatment arms: annual screening with CA125 as the primary test and ultrasound as a secondary test, an ultrasound group and a control group with no screening. ${ }^{21}$ All the women will be followed up for 7 years. At the time of writing, patient enrolment and randomisation have been completed and results are expected in 2011. Although the primary endpoint of the study is ovarian cancer mortality,

Table 1 Criteria for defining high-risk families in the UK Familial Ovarian Cancer Screening Study (UKFOCSS)22

1 Two or more first-degree relatives with ovarian cancer regardless of age.

2 One first-degree relative with ovarian cancer and one first-degree relative with breast cancer below the age of 50 years.

3 One first-degree relative with ovarian cancer and two first-degree relative with breast cancer below the age of 60 years.

4 An individual with one of the ovarian cancer predisposing genes.

5 Three first-degree relatives with colorectal cancer with at least one diagnosed before the age of 50 years and at least one first-degree relative with ovarian cancer.

6 Criteria 1-3 are modified when paternal transmission is occurring. Families where affected relatives are related by second degree through an unaffected intervening male relative who has an affected sister. 
Table 2 Ovarian cancer screening: key points

- Ovarian cancer is the leading cause of death from gynaecological malignancy in the Western world.

- No precancerous lesions have been identified.

- Bimanual examination is of no value as a screening test.

- Transvaginal screening has a high sensitivity for ovarian cancer; however, the detection of benign lesions may lead to unnecessary operations.

- High CA125 levels may be caused by many benign and physiological conditions. Measuring CA125 is of more benefit when used as part of a multimodal strategy.

- Women in the general population should not be screened unless they are taking part in clinical trials.

- Although there is currently no evidence of any benefit, screening is currently recommended for high-risk women.

other issues such as health economics, patient compliance and psychological and physical mortality associated with screening will also be addressed.

In UKFOCSS, a single-arm prospective study, 5000 high-risk women have an annual TVS scan and CA125.22 Additional 4-monthly blood samples are analysed retrospectively for CA125 and novel tumour markers with the aim of deriving a familial risk of ovarian cancer (FROC) index similar to the ROC used in the general population. Criteria for defining high-risk families in UKFOCSS are summarised in Table 1.

The results of these trials will not only provide evidence for whether screening provides a survival benefit at an acceptable financial cost, but will also address other issues such as the optimal age for the commencement of screening, optimal screening intervals, physical and psychological morbidity, and acceptability.

\section{Conclusions}

Progress into the early detection and treatment of ovarian cancer has been hampered by the lack of precursor lesions and the uncertainty regarding the duration of the preclinical phase of the disease. However, our understanding of ovarian cancer progression and detection will be improved by the large randomised trials that are currently in progress. Until these data become available, women in the general population should not be screened unless they are taking part in clinical trials. For high-risk women, screening is currently recommended as part of ongoing research, but these women need to be counselled that there is, as yet, no evidence of any benefit (Table 2).

Statements on funding and competing interests

Funding. None identified.

Competing interests. None identified.

\section{Acknowledgements}

The author wishes to thank Professor Ian Jacobs and Dr Usha Menon for their contribution in the field of ovarian cancer screening and their support.

\section{References}

1 CancerStats reports. Cancer Research UK, London, UK. http://info.cancerresearchuk.org/cancerstats/reports/ [Accessed 2 June 2006].

2 Ries LAG, Kosary CL, Hankey BF, Miller BA, Edwards BK (eds). SEER Cancer Statistics Review: 1973-1996. Bethesda, MD: National Cancer Institute, 1999

3 Jacobs I. Overview - progress in screening for ovarian cancer. In Ovarian Cancer 5, Sharp F, Blackett T, Berek J, Bast R (eds). Oxford, UK: Isis Medical Media: Oxford, 1998.

4 Surveillance Epidemiology and End Results (SEER) Program of the National Cancer Institute, US National Institutes of Health, Bethesda, MD, USA. http://seer.cancer.gov/ [Accessed 2 June 2006].

5 Schildkraut JM, Thompson WD. Familial ovarian cancer: a population-based case-control study. Am J Epidemiol 1988; 128: 456-466.

6 Antoniou A, Pharoah PD, Narod S, Risch HA, Eyfjord JE, Hopper JL, et al. Average risks of breast and ovarian cancer associated with BRCA1 or BRCA2 mutations detected in case series unselected for family history: a combined analysis of 22 studies. Am J Hum Genet 2003; 72: 1117-1130.

7 Modugno F. Ovarian cancer and high-risk women - implications for prevention, screening, and early detection. Gynecol Oncol 2003; 91: $15-31$.

8 Andolf E, Svalenius E, Astedt B. Ultrasonography for early detection of ovarian carcinoma. Br J Obstet Gynaecol 1986; 93: 1286-1289.

9 Campbell S, Bhan V, Royston P, Whitehead MI, Collins WP. Transabdominal ultrasound screening for early ovarian cancer. $B M J$ 1989; 299(6712): 1363-1367.

10 Karlan BY. The status of ultrasound and color Doppler imaging for the early detection of ovarian carcinoma. Cancer Invest 1997; 15: 265-269.

11 DePriest PD, Gallion HH, Pavlik EJ, Kryscio RJ, van Nagell JR Jr. Transvaginal sonography as a screening method for the detection of early ovarian cancer. Gynecol Oncol 1997; 65: 408-414.

12 van Nagell JR Jr, DePriest PD, Reedy MB, Gallion HH, Ueland FR, Pavlik EJ, et al. The efficacy of transvaginal sonographic screening in asymptomatic women at risk for ovarian cancer. Gynecol Oncol 2000; 77: $350-356$

13 Jacobs I, Bast RC Jr. The CA 125 tumour-associated antigen: a review of the literature. Hum Reprod 1989; 4: 1-12.

14 Sjovall K, Nilsson B, Einhorn N. The significance of serum CA 125 elevation in malignant and nonmalignant diseases. Gynecol Oncol 2002; 85: 175-178.

15 Einhorn N, Sjovall K, Knapp RC, Hall P, Scully RE, Bast RC Jr, et al. Prospective evaluation of serum CA 125 levels for early detection of ovarian cancer. Obstet Gynecol 1992; 80: 14-18.

16 Skates S, Pauler DK, Jacobs IJ. Screening based on the risk of cancer calculation from Bayesian hierarchical change-point and mixture models of longitudinal markers. I Am Stat Assoc 2001: 96: 429-439.

17 Jacobs IJ, Skates SJ, MacDonald N, Menon U, Rosenthal AN, Davies AP, et al. Screening for ovarian cancer: a pilot randomised controlled trial. Lancet 1999; 353(9160): 1207-1210.

18 Sato S, Yokoyama Y, Sakamoto T, Futagami M, Saito Y. Usefulness of mass screening for ovarian carcinoma using transvaginal ultrasonography. Cancer 2000; 89: 582-588.

19 Olivier RI, Lubsen-Brandsma MA, Verhoef S, van Beurden M. CA125 and transvaginal ultrasound monitoring in high-risk women cannot prevent the diagnosis of advanced ovarian cancer. Gynecol Oncol 2006; 100: 20-26.

20 Hasson MA, Fagerstrom RM, Kahane DC, Walsh JH, Myers MH, Caughman C, et al; Prostate, Lung, Colorectal and Ovarian Cancer Screening Trial Project Team. Design and evolution of the data management systems in the Prostate, Lung, Colorectal and Ovarian (PLCO) Cancer Screening Trial. Control Clin Trials 2000; 21(6 Suppl): 329S-348S.

21 UK Collaborative Trial of Ovarian Cancer Screening (UKCTOCS). http://www.ukctocs.org.uk [Accessed 2 June 2006].

22 National Cancer Research Network Trials Portfolio. UK Familial Ovarian Cancer Screening Study (UKFOCSS). http://www.ncrn. org.uk/portfolio/data.asp?ID=1069 [Accessed 2 June 2006]

\section{ERRATUM}

'Economic evaluation. Part 1: Introduction to the concepts of economic evaluation in health care', Emma McIntosh, Ramon Luengo-Fernandez, J Fam Plann Reprod Health Care 2006: 32(2): 107-112

Due to a typesetting error on page 107, the title of Box 1 was incorrect. The correct title is: Box 1: Glossary of terms.

The Journal wishes to apologise for this inadvertent error and for any inconvenience caused to readers or to the authors of the article in question. 